\title{
Understanding a key gene for thermosensitive genic male sterility in rice
}

\author{
FAN YouRong \& ZHANG Qifa* \\ National Key Laboratory of Crop Genetic Improvement and National Centre of Plant Gene Research, Huazhong Agriculture University, \\ Wuhan 430070, China
}

Received September 29, 2014; accepted October 8, 2014; published online October 17, 2014

Citation: $\quad$ Fan YR, Zhang QF. Understanding a key gene for thermosensitive genic male sterility in rice. Sci China Life Sci, 2014, 57: 1241-1242, doi: 10.1007/s11427-014-4763-6

Recently, a joint research team led by Chuxiong Zhuang of South China Agricultural University and Xiaofeng Cao of Institute of Genetics and Development Biology, Chinese Academy of Sciences published their work on the cloning and molecular characterization of the gene thermosensitive genic male sterile 5 (tms5) in rice in Nature Communications [1]. This is the result of a long-term collaboration representing an important advance in male sterility research in crops.

Rice (Oryza sativa L.) is one of the most important food crops in the world. Hybrid rice has significantly increased the yield level compared to inbred varieties. In the past decades, China has been energetically developing and popularizing hybrid rice to meet the food demand of the growing population. The three-line system and two-line system were the two approaches widely used in hybrid rice breeding. The three-line system is composed of a male-sterile line, a restorer line and a maintainer line, while in the two-line system the male-sterile line also serves as the maintainer line for the propagation of male sterile seeds. The sterile male-sterile line used in two-line system becomes fertile under particular growth environmental conditions, which greatly simplifies the process of hybrid breeding and saves tremendous cost in seed production. Photoperiod-sensitive genic male sterile lines (PGMS) and thermo-sensitive genic male sterile lines (TGMS) have been the two main types used in two-line hybrid rice breeding. As the name implies,

*Corresponding author (email: qifazh@mail.hzau.edu.cn) the fertility of PGMS and TGMS are sensitive to photoperiod and temperature respectively. Specifically, it is sterile under restrictive conditions (long-day for PGMS and high temperature for TGMS) and fertile under permissive conditions (short-day for PGMS and low temperature for TGMS). Since 1993, two-line hybrid rice has been planted on a gradually increasing scale, which now occupies a large rice area in China.

Recently, the mechanisms of the major types of cytoplasmic male sterility and fertility restoration systems used in the three-line systems in rice have been elucidated [2]. But the genes controlling genic male sterility in the two-line system have not been cloned until the work by Ding et al. [3] who mapped and cloned a long non-coding RNA LDMAR (long-day-specific male-fertility-associated RNA) regulating photoperiod-sensitive genic male sterility in rice. The mutation of a single nucleotide between the wild type Nongken 58 and PGMS Nongken 58S increased the methylation level in the promoter region of $L D M A R$, which suppressed the transcription of $L D M A R$ under long-day conditions, resulting in male sterility. Soon, Zhou et al. [4] also reported their work on the same locus and found the single nucleotide polymorphism (SNP) located in a 21-nucleotide small RNA osa-smR5864w produced by LDMAR. They speculated that the SNP may lead to loss-of-function of osa-smR5864m (the mutant small RNA corresponding to osa-smR5864w), causing PGMS and TGMS in the japonica and indica lines respectively.

So far, a number of genetic loci for thermo-sensitive 
genic male sterility had been identified in rice, such as tms 1 , tms2, tms3(t), tms4, tms5, tms6(t), TGMS, tms6, tms9, tms $9-1$ and rtms1. Genetic analysis indicated that the TGMS genes at these loci are all recessive. In the work by Zhou et al. [4], transformation of their identified candidate gene Os02g0214300 of TMS5 to TGMS lines AnS-1 and ZhuS1 restored the fertility of the transgenic plants at restrictive temperature. They found that TMS5 encodes a short form ribonuclease $\mathrm{Z}$ (RNase $\mathrm{Z}^{\mathrm{S}}$ ) referred to as RNase $\mathrm{Z}^{\mathrm{S} 1}$, and a C-to-A transition at position 71 of tms 5 transcript created a premature stop codon of RNase $Z^{\mathrm{S} 1}$. There was no difference in the RNase $Z^{S 1}$ expression at the mRNA level and protein level under the permissive and restrictive temperature, which indicated that the RNase $Z^{\mathrm{S} 1}$ is temperature insensitive and some other factors might be involved in the process of thermo-sensitive genic male sterility mediated by RNase $Z^{S 1}$. Next, they tested the RNase $Z^{S 1}$ endonuclease activity and detected that RNase $Z^{S 1}$ processed the $3^{\prime}$ end of tRNAs in vitro but not in vivo. Resorting to the analysis of whole genome microarray and RNA-seq, the researchers identified three mRNAs named $U b_{L 40} 1, U b_{L 40} 2$ and $U b_{L 40} 4$, whose genes belong to the conserved ubiquitin-60S ribosomal protein L40 family $\left(U b_{L 40}\right)$, accumulating at higher level in the young panicles of tms 5 plants at high temperature than at low temperature. Overexpression of $U b_{L 40} l$ and $U b_{L 40} 4$ in wild type $\mathrm{ZH} 11$ reduced the pollen fertility, while the $U b_{L 40} l$ and $U b_{L 40} 4$ RNAi lines in plants containing the tms 5 gene showed increased pollen fertility at restrictive temperature. The mRNAs of the three $U b_{L 40}$ genes could be processed into multiple fragments by RNase $Z^{S 1}$. The au- thors thus proposed a working model for tms5-mediated TGMS. In the TGMS line, the $U b_{L 40}$ mRNA was expressed at high level at high temperature but could not be processed by dysfunctional RNase $\mathrm{Z}^{\mathrm{S} 1}$, resulting in excessive accumulation of this mRNA leading to male sterility, while there was a relatively low level of $U b_{L 40}$ mRNA expression at low temperature thus increasing male fertility. By contrast, in wild type, $U b_{L 40}$ mRNA transcript was kept at low level by RNase $\mathrm{Z}^{\mathrm{S} 1}$ processing regardless of the temperature conditions.

This is the first TGMS gene cloned from a useful germplasm for hybrid rice production. The result may help future application in developing male sterile lines in hybrid rice breeding, in addition to enhanced understanding of male fertility development in plants.

1 Zhou H, Zhou M, Yang YZ, Li J, Zhu LY, Jiang DG, Dong JF, Liu QJ, Gu LF, Zhou LY, Feng MJ, Qin P, Hu XC, Song CL, Shi JF, Song XW, Ni ED, Wu XJ, Deng QY, Liu ZL, Chen MS, Liu YG, Cao XF, Zhuang CX. RNase $Z^{S 1}$ processes $U b_{L 40}$ mRNAs and controls thermosensitive genic male sterility in rice. Nat Commun, 2014, 5: 4884-4892

2 Chen L, Liu Y. Male sterility and fertility restoration in crops. Annu Rev Plant Biol 65: 579-606

3 Ding JH, Lu Q, Ouyang YD, Mao HL, Zhang PB, Yao JL, Xu CG, Li $\mathrm{XH}$, Xiao JH, Zhang QF. A long noncoding RNA regulates photoperiod-sensitive male sterility, an essential component of hybrid rice. Proc Natl Acad Sci USA, 2012, 109: 2654-2659

4 Zhou H, Liu QJ, Li J, Jiang DG, Zhou LY, Wu P, Lu S, Li F, Zhu LY, Liu ZL, Chen LT, Liu YG, Zhuang CX. Photoperiod- and thermo-sensitive genic male sterility in rice are caused by a point mutation in a novel noncoding RNA that produces a small RNA. Cell Res, 2012, 22: 649-660

Open Access This article is distributed under the terms of the Creative Commons Attribution License which permits any use, distribution, and reproduction in any medium, provided the original author(s) and source are credited. 